\title{
Longitudinal changes in uterine, umbilical and fetal MCA Doppler indices in late onset small fetuses (Doppler in small fetuses)
}

\author{
Abdelhassib Salah Abdelhassib Saad ${ }^{1}$, Medhat Esam Eldeen Helmy ${ }^{1}$, \\ Ahmed Nabil Abdelhameed Eissa ${ }^{1}$, Mohamed Momtaz Mohamed Awad ${ }^{2}$, \\ Hassan Mostafa Ismael Gahfar ${ }^{2}$, Wael Gaber Elsyed El Damaty ${ }^{1}$ \\ ${ }^{1}$ Obstetrics and Gynecology Department, Faculty of Medicine, Menoufia University, sheben el kom, Egypt \\ ${ }^{2}$ Obstetrics and Gynecology Department, Faculty of Medicine, Cairo University, Cairo, Egypt
}

\section{Email address:}

Abdelhassebsalah@yahoo.com (A. S. A. Saad), hassangaafar@hotmail.com (H. M. Gaafar)

\section{To cite this article:}

Abdelhassib Salah Abdelhassib Saad, Medhat Esam Eldeen Helmy, Ahmed Nabil Abdelhameed Eissa, Mohamed Momtaz Mohamed Awad, Hassan Mostafa Ismael Gahfar, Wael Gaber Elsyed El Damaty. Longitudinal Changes in Uterine, Umbilical and Fetal MCA Doppler Indices in Late Onset Small Fetuses (Doppler in Small Fetuses). American Journal of Health Research.

Vol. 2, No. 5, 2014, pp. 222-228. doi: 10.11648/j.ajhr.20140205.11

\begin{abstract}
Objectives: To determine the longitudinal trends and rates of conversion of normal to abnormal uterine (UtA), umbilical (UA) and middle cerebral artery (MCA) Doppler velocimetry throughout the third trimester in late-onset smallfor-gestational-age (SGA) fetuses. Background: The finding of a small abdominal circumference should stimulate the sonographer to consider three possible: wrong date, normal small or abnormal small fetus. Methods: UtA, UA and MCA Doppler velocimetry was serially performed in a cohort of singleton consecutive late-onset SGA fetuses with normal Doppler values at diagnosis. The rate of conversion of normal to abnormal Doppler values was evaluated by survival analysis. Longitudinal trends were modeled by means of multilevel analysis. Results: A total number of study group included 150 SGA fetuses with normal Doppler upon diagnosis. Mean gestational age at inclusion and at delivery was 33.23 (SD 1.39) and 38.14 (SD 1.02) weeks, respectively. the longitudinal trends in UA PI and UtA PI showed no changes in contrast to MCA and CPR demonstrated a clear and progressive decrease in values from 7th week of follow up (37week of gestation) to delivery., mean MCA-PI 1.67 (SD 0.16) while mean CPR-PI 2.06 (SD 0.71) in all weeks of follow up for both. Conclusion: Late-onset SGA fetuses with normal Doppler velocimetry upon diagnosis show progression from 37 weeks' gestation with worsening CPR followed by a decrease in MCA-PI.
\end{abstract}

Keywords: Doppler Ultrasonograph, Umbilical Artery Doppler, Middle Cerebral Artery Doppler, Cerebro Placental Ratio, Late Onset Small for Gestational Age

\section{Introduction}

The terms small-for-gestational-age (SGA) and intrauterine growth restriction (IUGR) have often been used interchangeably, but not all small fetuses are growth restricted. Most instances of true growth restriction correspond with cases of placental insufficiency ${ }^{(1)}$.

In early-onset IUGR (i.e. onset before 34 weeks' gestation), the clinical standard for identifying SGA fetuses with IUGR is umbilical artery (UA) Doppler ultrasonography, and IUGR so defined is associated with adverse perinatal and neurodevelopmental outcomes ${ }^{(2)}$.
However, in late-onset IUGR the presence of placental insufficiency is often not reflected in UA Doppler. Although for a long time SGA with normal UA Doppler near term was considered a benign non pathological condition, it is now widely accepted that such fetuses include a significant proportion of late-onset forms of IUGR with increased risk of adverse perinatal outcome ${ }^{(3)}$.

Recent studies suggest that Doppler ultrasound in other vascular territories, such as brain or uterine arteries (UtAs), could greatly improve the detection of late-onset IUGR that is missed by UA Doppler ${ }^{(4)}$.

Fetal growth restriction (FGR) complicates up to $8 \%$ of 
pregnancies and carries high perinatal morbidity and mortality. Infants born as small-for-gestational-age (SGA) also present a greater risk for cardiovascular disease during adulthood $^{(5)}$.

Placental function evaluation by umbilical artery (UA) Doppler velocimetry is the clinical standard for identifying early-onset intrauterine growth restriction (IUGR) and there is evidence that its use in these pregnancies improves a number of obstetric care outcomes and reduces perinatal deaths ${ }^{(6)}$.

Since UA Doppler cannot be relied on for the identification of late-onset SGA fetuses at risk for adverse perinatal outcome, other vascular territories have been proposed, including the uterine artery (UtA) and fetal middle cerebral artery (MCA). Abnormal UtA Doppler has been shown to be comparable with UA Doppler as a predictor of adverse outcome in late-onset IUGR. On the other hand, a reduced pulsatility index in the MCA is also associated with poorer perinatal outcome, and with an increased risk of abnormal neurodevelopment. Finally, the cerebroplacental ratio (CPR), which combines the pulsatility index (PI) of the MCA and UA, has been shown to be more sensitive to hypoxia than its individual components in animal and clinical models, and correlates better with adverse outcome ${ }^{(7)}$.

\section{Material and Methods}

The study was conducted in fetal medicine unit and high risk unit at Kasr Al Ainy hospital in obstetrics and gynecology department, Cairo University and out patient's clinic in obstetrics and gynecology department, Menoufiya university hospital. Patients were recruited in the period from June 2011 to December 2013. The study group consisted of 150 pregnant patients diagnosed with late onset small for gestational age (SGA) fetuses throughout the third trimester scan.

\subsection{Inclusion Criteria}

1. Patients with suspected SGA singleton fetuses (AC or $\mathrm{EFW}<10$ th percentile but normal interval growth) at routine third trimester ultrasonography scan (3036 weeks gestation), patients sure of dates with regular cycles or had 1 st trimester CRL measurement.

2. Normal Doppler indices, mean uterine artery pulsatity index (UtA-PI) <95th centile, umbilical artery pulsatity index (UA-PI) $<95$ th centile, middle cerebral pulsatity index (MCA-PI $>5$ th centile and cerebro placental ratio $(\mathrm{CPR})>5$ th centile.

\subsection{Exclusion Criteria}

1. Postnatal confirmation of a birth weight $>10$ th centile.

2. Presence of structural or chromosomal anomalies.
3. Patients presented with any medical disorders that may cause intrauterine growth retardation (IUGR) as pre-eclampsia.

All patients in the study underwent uniform antenatal assessment protocol that included a four component history including dating of pregnancy, examination, biophysical profile score (BPP), and Doppler indices (RUtA- PI, LUtAPI and mean UtA-PI, UA-PI, MCA-PI, and CPR), history included age, parity, past history, smoking and spontaneous or induced pregnancy. Dating of pregnancy was calculated by 1 st trimester CRL measurement or by date of LMP, examination included general and physical examination of the abdomen by inspection and palpation (fundal level and $\mathrm{SFH}$ ).

In the study group Doppler velocimetry was performed weekly and non stress test and BPP were conducted either twice weekly or more according to the severity of the condition and all cases had Doppler examination within 7 days of delivery

Ultrasound equipment capable of high resolution grayscale, pulsed wave and color Doppler modes was used, (accuvix XQ, Medison, Seol, Korea),(Volluson Pro V 370, General Electric, USA) and (Sonata plus,Mindery,China) using 3.5 MHZ covex probe.

Pulsed Doppler parameters was calculated automatically from 3 or more consecutive waveforms, with angel of insonation $<30^{\circ}$ as possible.

The uterine arteries were examined transabdominally; the PI of the left and right arteries were measured, and the mean index was calculated. Doppler flow spectra were obtained from the UA at the midsection of the umbilical cord and the distal portion of the MCA by methods that have been previously described. The CPR was calculated as the ratio of the MCA-PI to the UA-PI.

\subsection{Timing of the Delivery}

As clear guidelines supported by strong evidence are not available protocols of management should be based on the available knowledge and clinical judgments. (Table 1)

Neonatal assessment by staff of pediatrics in the form of:

- Wight of the neonate. (excluded if a birth weight $>10$ th centile)

- $\quad$ APGAR score at1 and $5 \mathrm{~m}$ were recorded.

- Neonate needs incubator or not.

All Doppler parameters were transformed into Z-values according to normative references. The longitudinal changes were analyzed by Kaplan-Meier survival analysis, in which the endpoint was defined as an abnormal Doppler value (MCA-PI and $\mathrm{CPR}<5$ th centile, UAand UtAPIs >95th centile)22,27. The McNemar test was used to compare paired group proportions. Statistical and survival analyses were performed using the Statistical Package for Social Sciences software (SPSS 15.0, SPSS Inc., Chicago, IL, USA). 
Table 1. Indication of termination of pregnancy depend on BPP score, NST and Doppler indices

\begin{tabular}{|c|c|c|}
\hline Gestational age & Management & Recommendation \\
\hline$<34$ weeks & $\begin{array}{l}\text { Doppler (UA PI) and fetal tests } \\
\text { (BPP and NST) }\end{array}$ & $\begin{array}{l}\text { Consider corticosteroids administration. } \\
\text { If EDV present and good fetal tests follow up twice a week. } \\
\text { If AREDV daily UA and DV Doppler, delivery if abnormal DV Doppler and/or } \\
\text { fetal tests. }\end{array}$ \\
\hline 34-37weeks & $\begin{array}{l}\text { Doppler (UA PI, MCA PI and } \\
\text { CPR PI) and fetal tests (BPP and } \\
\text { NST) }\end{array}$ & $\begin{array}{l}\text { If UA PI }>95^{\text {th }} \text { but EDV present, brain sparing effect (MCA PI and CP R PI } \\
<5^{\text {th }} \text { centil) and good fetal tests follow up twice a week and consider delivery } \\
\text { in abnormal fetal tests. } \\
\text { If AREDV delivery is advisable even with good fetal tests. }\end{array}$ \\
\hline$>37$ weeks & $\begin{array}{l}\text { Doppler (UA PI, MCA PI and } \\
\text { CPR PI) and fetal tests (BPP and } \\
\text { NST) }\end{array}$ & $\begin{array}{l}\text { - Offer delivery by } 37 \text { weeks } \\
\text {-Recommend delivery if } \mathrm{MCA} \mathrm{PI}<5^{\text {th }} \text { centile }, \mathrm{CPR} \mathrm{PI}<5^{\text {th }} \text { centil, UA PI }>95^{\text {th }} \\
\text { and/or } \mathrm{BPP}<6 \\
\text { Consider induction of labor at } 40 \pm \text { week even with nrmal Doppler indices }\end{array}$ \\
\hline
\end{tabular}

\section{Results}

During the study period a total of 160 SGA fetuses fulfilled the inclusion criteria. Ten cases were excluded because eight cases had postnatal birth weight above the 10th centile and two cases had congenital heart diseases. Clinical data of 150 SGA fetuses of pregnant women includes maternal age, parity, BMI, smoking, past history, spontaneous or induced pregnancy. (Table 2)

The mean gestational age at inclusion was 33.23 (SD 1.39; range, 30.0-35.6) weeks and that at delivery was 38.14 (SD 1.02; range, 34.5-41.2) weeks. The median number of ultrasound scan was 7.62 (SD 1.91; range, 3-10) scan.

The proportion of abnormal Doppler variables before delivery (during follow up) and at the last examination within one week before delivery. Remarkably, the proportions of abnormal UA-PI ( 22 vs. $24 \%$; P value $>0.05$ ) were not significantly difference between two examination. However, the proportions of abnormal MCA-PI (20 vs. $44 \%$; P value $<0.001$ ) and abnormal CPR (32 vs. $54.7 \%$; P value $<0.001)$ were significantly different between these two examinations. Before delivery, the proportion of abnormal UA-PI was significantly lower than the proportion of abnormal MCA-PI ( 24 vs. $44 \%$; P $<0.001$ ) and abnormal CPR (24 vs. $54.7 \%$; P $<0.001$ ). Also, the proportion of abnormal MCA-PI and CPR differed significantly (44 vs. $54.7 \% ; \mathrm{P}=0.002$ ). (Table 3 ) (Figure1).
Table 2. Distribution of the studied patients regarding their clinical characteristics:

\begin{tabular}{lll}
\hline & $(\mathbf{n}=\mathbf{1 5 0})$ & $\mathbf{\%}$ \\
\hline Age (Y) & $\mathbf{3 0 . 4 6} \pm \mathbf{6 . 8 5}(\mathbf{1 8 - 4 5 )}$ \\
Mean \pm SD; Range & & \\
\hline Parity & 81 & 54.0 \\
P0 & 24 & 16.0 \\
P1 & 21 & 14.0 \\
P2 & 9 & 6.0 \\
P3 & 9 & 6.0 \\
P4 & 3 & 2.0 \\
P5 & 3 & 2.0 \\
P6 & & \\
BMI & 39 & 26.0 \\
Underweight & 75 & 50.0 \\
Normal & 27 & 18.0 \\
Overweight & 9 & 6.0 \\
Obese & & \\
Smoking (passive or active) & 25 & 16.6 \\
Yes & 125 & 83.3 \\
No & & \\
Past history & 27 & 56.7 \\
Yes & 123 & \\
No & & \\
Spontaneous or Induced pregnancy & & \\
Spontaneous pregnancy & & \\
Induced pregnancy & & \\
\hline & & \\
\hline
\end{tabular}

Table 3. Distribution of the studied patients regarding their UA, MCA and CPR Doppler before delivery (during follow up) and at delivery (one week before delivery):

\begin{tabular}{|c|c|c|c|c|c|}
\hline & \multicolumn{2}{|c|}{ Before delivery } & \multicolumn{2}{|c|}{ At delivery } & \multirow{2}{*}{ P value } \\
\hline & no & $\%$ & no & $\%$ & \\
\hline \multicolumn{6}{|l|}{ UA } \\
\hline Abnormal $\left(\mathrm{PI}>95^{\text {th }}\right.$ centil $)$ & 33 & 22.0 & 36 & 24.0 & 0.549 \\
\hline Normal & 117 & 78.0 & 114 & 76.0 & \\
\hline \multicolumn{6}{|l|}{ MCA } \\
\hline Abnormal $\left(\mathrm{PI}<5^{\text {th }}\right.$ centil $)$ & 30 & 20.0 & 66 & 44.0 & $<0.001$ (HS) \\
\hline Normal & 120 & 80.0 & 84 & 56.0 & \\
\hline \multicolumn{6}{|l|}{ CPR } \\
\hline Abnormal $\left(\mathrm{PI}<5^{\text {th }}\right.$ centil $)$ & 48 & 32 & 82 & 54.7 & $<0.001$ (HS) \\
\hline Normal & 102 & 68 & 68 & 45.3 & \\
\hline
\end{tabular}




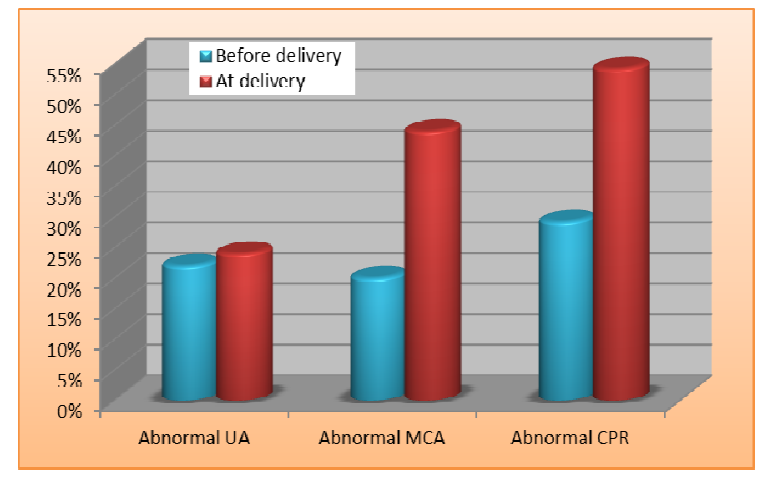

Fig 1. Distribution of the studied patients regarding their UA, MCA and $C P R$ Doppler before delivery (at diagnosis) and at delivery (one week before delivery):
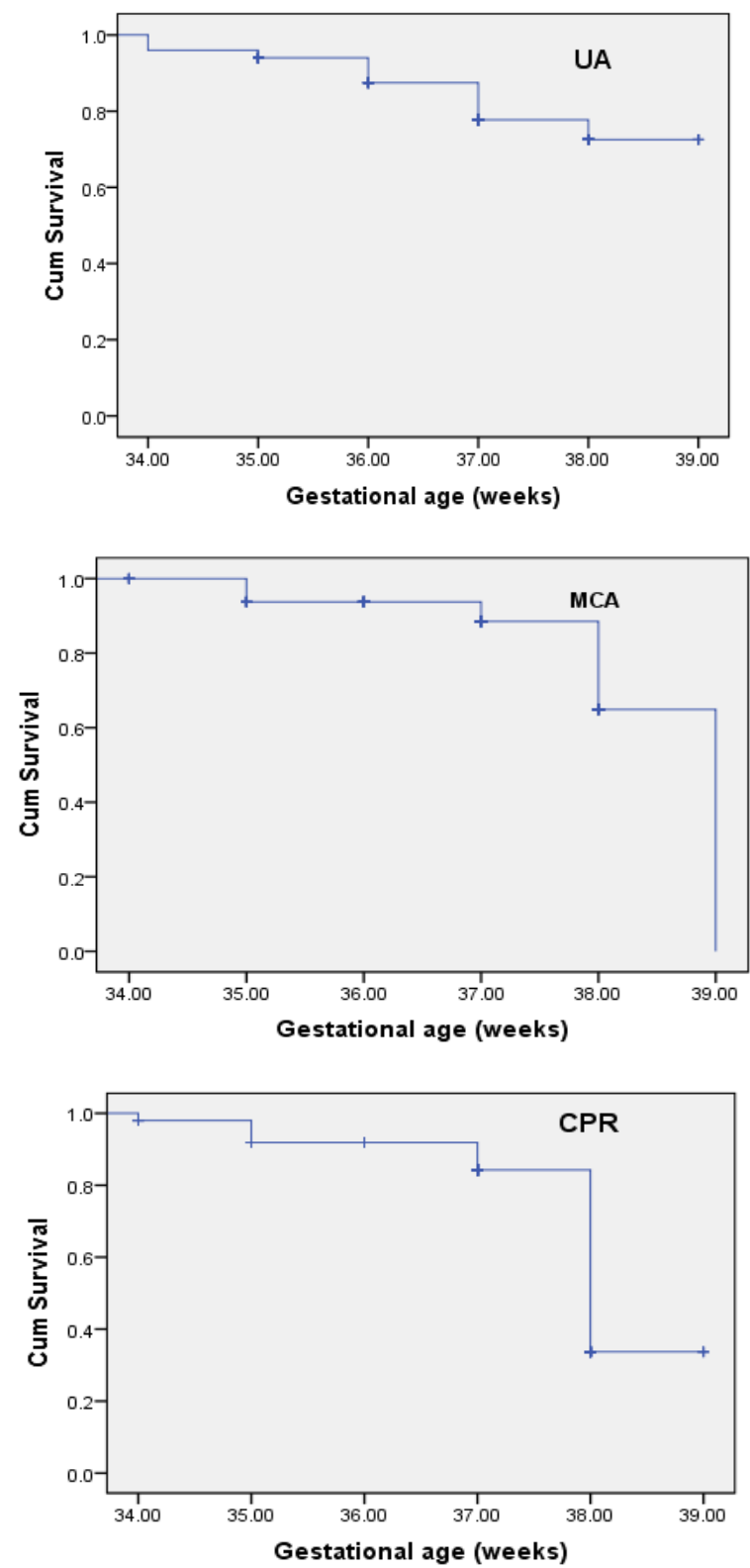

Fig 2. Kaplan-Meier curve for Proportion of fetuses with normal UA, $M C A$ and $C P R$ before delivery in relation to gestational age.
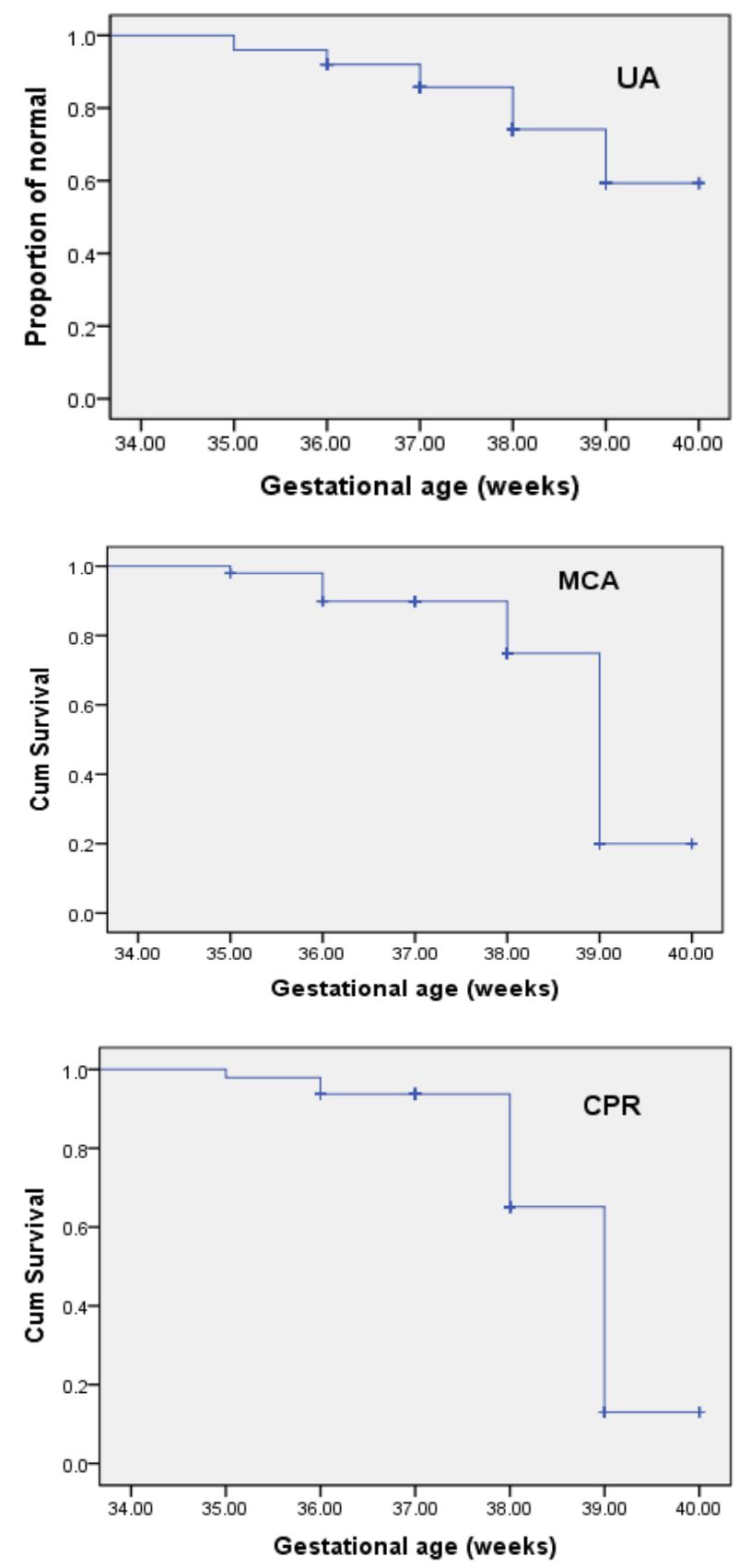

Fig 3. Kaplan-Meier curve for Proportion of fetuses with normal UA, $M C A$ and $C P R$ at delivery in relation to gestational age.

The survival graph for the Doppler parameters throughout the study period, plotted against gestational age, which could be interpreted as the remaining proportion of normal Doppler at each week of gestational age for each of the parameters. The remaining proportions $(95 \%$ confidence interval (CI)) of cases with normal UAPI ,MCA and CPR before delivery during follow up were $78 \%, 80 \%$ and $68 \%$ and at delivery (within one week of delivery) were $76 \%, 56 \%$ and $45.3 \%$ respectively.(table 4)(Fig 2,3)

The longitudinal trends of UA-PI, UtA- PI, MCA-PI and CPR-PI from enrollment to delivery. UA-PI 1.06 (SD 0.35) 
and mean UtA-PI 0.99 (SD 0.29) showed slightly increasing trend, while MCA-PI 1.67 (SD0.16) and CPRPI 2.06 (SD 0.71) demonstrated a clear and progressive decrease in values from inclusion to delivery. (Fig 4)
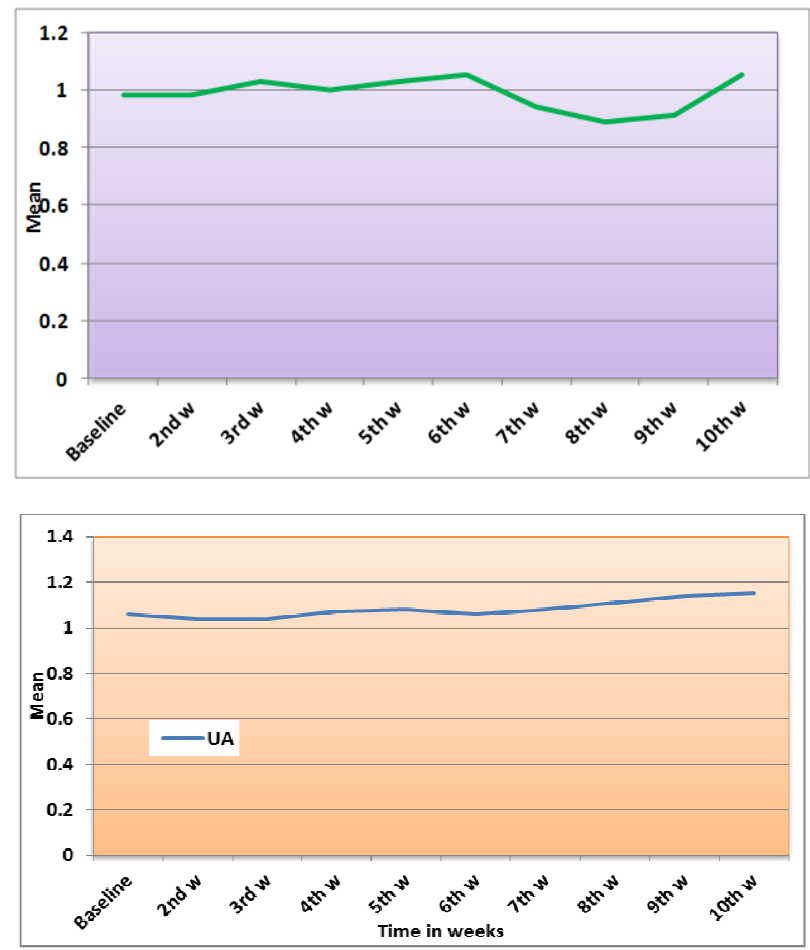

Fig 4. The longitudinal trends of Doppler parameters (in Z-values) during the study period of UA-PI and UtA-PIfrom enrollment to delivery.

\section{Discussion}

Fetal abdominal circumference (AC) or estimated fetal weight $(\mathrm{EFW})<10$ th centile can be used to diagnose a SGA fetus. Biophysical tests, including amniotic fluid volume, cardiotocography (CTG) and biophysical scoring are poor at diagnosing a small or growth restricted fetus ${ }^{(8)}$.

One of the key points in this study that is different from many previous studies is that we diagnosed the SGA status of the infant not by antenatal ultrasound assessment, but also by birth weight measured after birth $<10$ th centil. This method would eliminate possible errors in birth weight measurement.

Despite the high prevalence of late-onset SGA, little is known about its Doppler evolution at the end of pregnancy. This is the first study to describe the longitudinal changes in uteroplacental and fetal brain hemodynamics focusing on late-onset SGA fetuses.

Evidence shows that umbilical artery Doppler can be used to distinguish between the high-risk small fetus that is truly growth-restricted and the lower-risk small fetus ${ }^{(9)}$.

Our study together with the findings of Oros et al., 2011 $1^{(7)}$ shows that, on average, fetoplacental Doppler indices remain virtually unchanged from inclusion to delivery and consequently the proportion of cases progressing to abnormal Doppler velocimetry in the UA PI and mean UtA
PI is negligible, except in this our study L UtA PI shows significant changes in most weeks of follow up (Fig 4), while MCA-PI and CPR demonstrated a clear and progressive decrease in values from inclusion to delivery (Fig 5).
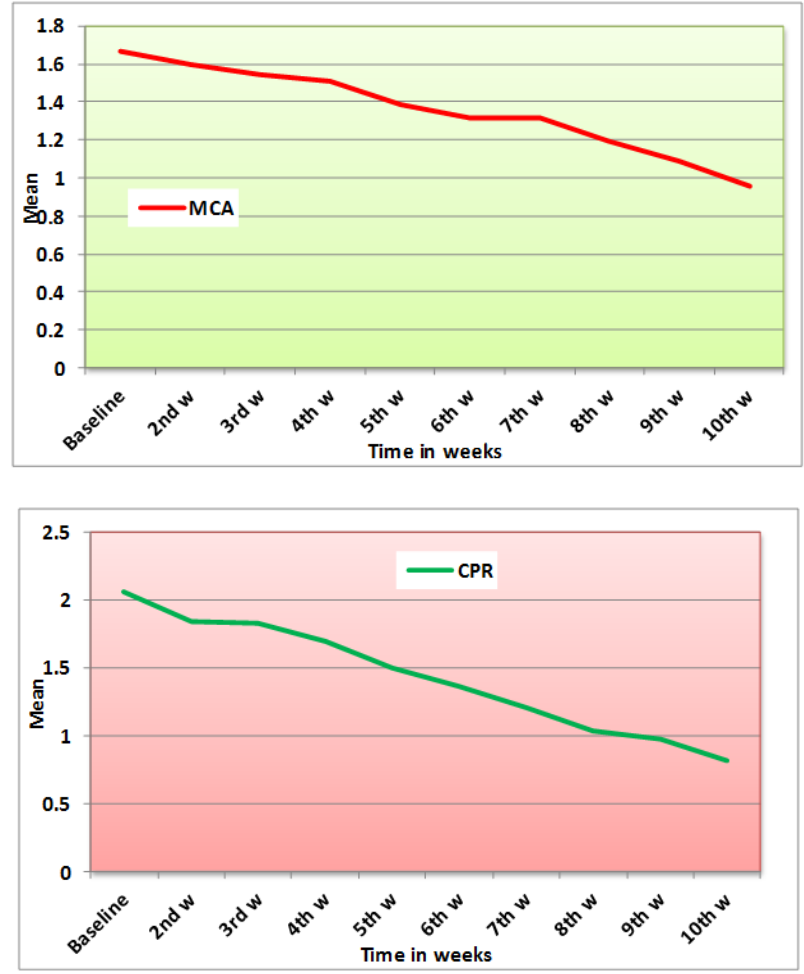

Fig 5. The longitudinal trends of Doppler parameters (in Z-values) during the study period of MCA-PI and CPR-PI from enrollment to delivery

The inclusion of UtA Doppler velocimetry is based on the evidence that an impaired placental flow negatively affects fetal growth. The finding of a persistent high resistance in the third trimester may contribute to the identification of fetuses undergoing intrauterine growth restriction (IUGR), among the SGA group ${ }^{(10)}$.

However, recent evidence strongly suggests that UA Doppler is not a reliable sign of placental insufficiency in mild forms of fetal growth restriction near or at term. A proportion of term SGA fetuses with normal UA PI have a higher risk of abnormal neonatal neurobehavioral performance with poorer outcome ${ }^{(11)}$.

In normal pregnancies the diastolic component in the cerebral arteries is lower than in the umbilical arteries at any gestational age. Therefore, the cerebro-vascular resistance remains higher than the placental resistance and the MCA/UA ratio is greater than 1 and the index becomes less than 1 if the flow distribution is in favor of the brain in pathological pregnancies ${ }^{(12)}$

According to Gramellini et al., 1992 and Arduini et al., $1992^{(13,14)}$ assessment of MCA/UA PI index provide better information in predicting perinatal outcome when compared with umbilical or middle cerebral artery Doppler indices alone.

Middle cerebral artery (MCA) pulsed Doppler has long 
constituted the clinical standard for the diagnosis of brain redistribution. Up to $15 \%$ of term SGA has a reduced pulsatility index (PI) in the MCA, and this is associated with poorer perinatal outcome ${ }^{(11)}$.

Anterior cerebral artery (ACA) PI or the cerebroplacental ratio (CPR), have been proposed for clinical detection of brain redistribution in growthrestricted fetuses and in SGA fetuses with normal UA PI ${ }^{(15)}$.

On the other hand, average MCA-PI and, more markedly, CPR values worsen significantly from one week before and until delivery, with 20 and $32 \%$ before delivery and $44 \%$ and $54.7 \%$ at delivery of abnormal values, respectively. The study provides evidence to support the notion that uteroplacental Doppler is of little clinical value in SGA fetuses and that cerebral Doppler may constitute the most sensitive method for identifying cases at high risk of adverse perinatal outcome.

The survival graph for the Doppler parameters throughout the study period, plotted against gestational age, which could be interpreted as the remaining proportion of normal Doppler at each week of gestational age for each of the parameters. The remaining proportions $(95 \%$ confidence interval (CI)) of cases with normal UAPI and MCA-PI and CPR before delivery versus at delivery were UA ( 78 vs. $76 \%$; $\mathrm{P}=0.549)$, MCA ( 80 vs. $56 \% ; \mathrm{P}<0.001)$, CPR (68 vs. $45.3 \%$; $<<0.001)$. (table 2) while In Oros et al.,2012 ${ }^{(7)}$, The remaining proportions (95\% confidence interval (CI)) of cases with normal UtA PI, UA PI,MCA-PI and CPR were 98.6 (96-100), 94.5 (85.3-100), 85 (76.293.8) and 49.6\% (35.1-64.1), respectively. Cruz-Martinez et al.,2011 ${ }^{(11)}$ reported that At 37 weeks, the proportion of abnormal values was $10.8 \%$ (95\% confidence interval [CI],
4.1-17.4) for the MCA PI, 16.8\% (95\% CI, 8.7-24.9) for the CPR, $17.2 \%(95 \% \mathrm{CI}, 9.3-25.4)$ for the ACA PI, and $31.3 \%(95 \% \mathrm{CI}, 21.5-41.0)$ for the fractional moving blood volume ( FMBV).

This study has some limitations. Since abnormal UA Doppler is an indication for delivery for ethical reasons, the temporal relationship between the parameters studied may be biased. However, as abnormal UA Doppler indices were found in a small proportion of cases, the effect of this bias should be negligible. Any clinical study on the surveillance of fetal growth needs to be interpreted with caution because it can never be entirely blinded, and clinical management is influenced by the antenatal findings. However, clinicians attending the deliveries were unaware of the MCA, UtA and CPR Doppler status. Another limitation is that the study protocol did not include other Doppler parameters, such as ductus venosus or myocardial performance index, which may also have been informative.

\section{Conclusion}

Our study concludes that late-onset SGA fetuses with normal Doppler upon diagnosis show no changes in UA PI and UtA PI in contrast to a progression after 37 weeks of gestation with worsening of Doppler indices in all group study, UA PI in up to $24 \%$, MCA PI in $44 \%$ and CPR PI in up to $54 \%$ of cases is observed at last examination (at delivery). These findings discourage the use of UA as a single parameter for surveillance in late-onset SGA and support the use of the CPR as the most sensitive Doppler parameter for the clinical monitoring of these fetuses.

Table 4. Distribution of the studied patients regarding proportion of fetuses with normal UA, MCA and CPR before and at delivery:

\begin{tabular}{|c|c|c|c|c|c|c|}
\hline & \multicolumn{3}{|c|}{ Before delivery } & \multicolumn{3}{|c|}{ At delivery } \\
\hline & \multirow{2}{*}{$\%$} & Overall survival & \multirow{2}{*}{ SE } & \multirow{2}{*}{$\%$} & \multirow{2}{*}{$\begin{array}{l}\text { Overall survival } \\
\text { Mean }(95 \% \mathrm{CI})\end{array}$} & \multirow{2}{*}{ SE } \\
\hline & & Mean $(95 \%$ CI) & & & & \\
\hline UA & 78.0 & $38.27(38.05-39.50)$ & 0.11 & 76.0 & $39.07(38.83-39.80)$ & 0.14 \\
\hline MCA & 80.0 & $38.41(38.21-38.61)$ & 0.10 & 56.0 & $38.73(38.52-38.93)$ & 0.10 \\
\hline CPR & 68.0 & $37.99(37.78-38.20)$ & 0.10 & 45.3 & $38.64(38.46-38.82)$ & 0.09 \\
\hline
\end{tabular}

\section{References}

[1] Lee PA, Chernausek SD, Hokken-Koelega AC, Czernichow $P$; International Small for Gestational Age Advisory Board. International Small for Gestational Age Advisory Board consensus development conference statement: management of short children born small for gestational age, April 24October, 2001. Pediatrics 2003; 111: 1253-1261.

[2] Royal college of obstetrics and gynaecology (RCOG). Greentop guidelines. The investigation and management of the small-forgestational-age fetus. RCOG Press: London, 2002.

[3] Figueras F, Eixarch E, Meler E, Iraola A, Figueras J, Puerto B, Gratacos E. Small-for-gestational-age fetuses with normal umbilical artery Doppler have suboptimal perinatal and neurodevelopmental outcome. Eur $J$ Obstet Gynecol Reprod Biol 2008; 136: 34-38.

[4] Savchev S, Figueras F, Cruz-Martinez R, Illa M, Botet F, Gratacos E: Estimated weight centile as a predictor of perinatal outcome in small-for-gestational-age pregnancies with normal fetal and maternal Doppler indices. Ultrasound Obstet Gynecol 2012; 39: 299-303

[5] Pisaneschi S, Francesca A. L. Strigini, Angel M. Sanchez, Silvia Begliuomini, and et al. Compensatory Feto-Placental Upregulation of the Nitric Oxide System during Fetal Growth Restriction. PLoS One. 2012; 7(9): e45294.

[6] RCOG: Royal College of Obstetricians and Gynaecologists green-top guidelines (UK): the investigation and management of the small for- gestational-age fetus. http//:rcog.org.uk/files/rcogcorp/uploadediles/GT31SmallGestationalAgeFetus.pdf,2009. 
[7] Oros D, Figueras F, Cruz-Marteinz R, Meler E, M. Munmany $M$ and Gratacos E: Longitudinal changes in uterine, umbilical and fetal cerebral Doppler indices in lateonset small-for-gestational age fetuses, Ultrasound Obstet Gynecol 2011; 37: 191-195.

[8] RCOG: Royal College of Obstetricians and Gynaecologists green-top guidelines (UK): The investigation and management of the small for-gestational-age fetus. http//:rcog.org.uk/files/rcogcorp/Uploaded-files/GT31

SmallGestationalAgeFetus.pdf. (Green-top No31) 2nd Edition | February 2013, Minor revisions - January 2014

[9] Ursula F. Harkness, MD, MPH, Giancarlo Mari, MD. Diagnosis and management of intrauterine growth restriction. Clin Perinatol 2004; 31:743- 764

[10] Di Lorenzo G, Monasta L, Ceccarello M, et al. Third trimester abdominal circumference, estimated fetal weight and uterine artery doppler for the identification of newborns small and large for gestational age. European Journal of Obstetrics \& Gynecology and Reproductive Biology 166 (2013) 133-138.
[11] Cruz-Martinez R, Figueras F, Hernandez-Andrade E, Oros D,Gratacos E. Fetal brain Doppler to predict cesarean delivery for nonreassuring fetal status in term small-forgestational-age fetuses. Obstet Gynecol 2011; 117: 618-626.

[12] Arbeille P, Carles G, Chevillot M, Herve P, Perrotin F and Maulik D. Cerebral and umbilical Doppler in the prediction of fetal outcome. In: Maulik D and Zalud I (eds). Doppler ultrasound in obstetrics and gynecology.2005; 2nd ed. Springer-Verlag Berlin Heidelberg New York; 177-198.

[13] Gramellini D, Folli MC, Raboni S, Vadora E, Merialdi A.Cerebral-umbilical Doppler ratio as a predictor of adverse perinatal outcome. Obstet Gynecol 1992; 79: 416-420.

[14] Arduini D, Rizzo G, Boccolini MR, Romanini C, Mancuso S. Functional assessment of uteroplacental and fetal circulations by means of color Doppler ultrasonography. J Ultrasound Med 1992; 9: 249-53

[15] Turan OM, Turan S, Gungor S, Berg C, Moyano D, GembruchU, Nicolaides KH, Harman CR, Baschat AA. Progression of Doppler abnormalities in intrauterine growth restriction. Ultrasound Obstet Gynecol 2008; 32: 160-167. 